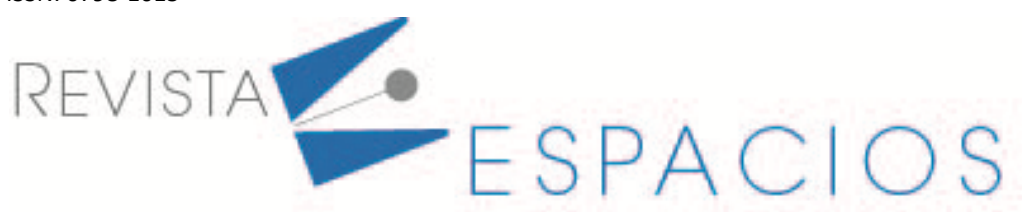

\title{
La política fiscal y su incidencia en la cultura tributaria del Ecuador
}

\section{Fiscal policy and its impact on the tax culture of Ecuador}

\author{
SIGUENZA, Sofía N. ${ }^{1}$ \\ SANDOVAL, Karen $\mathrm{S}^{2}$ \\ VIZCAÍNO, Alexandra M. ${ }^{3}$ \\ GIRON, Elizabeth A. ${ }^{4}$ \\ ERAZO, Sandra P. ${ }^{5}$ \\ JIMBO, Luis E. ${ }^{6}$
}

\section{Resumen}

La política fiscal y su incidencia en la cultura tributaria del Ecuador es una investigación de tipo documental que permitió determinar que la administración de la política fiscal se orienta en la generación de ingresos que son destinados a satisfacer las necesidades de la sociedad; pero que esta se ve afectada por la evasión fiscal, que es uno de los mayores fenómenos que afectan directamente el sistema tributario del Ecuador. El no pago intencional de las contribuciones no sólo erosiona los ingresos del Estado, sino que deteriora la estructura y economía de un país, causando un efecto dañino para la sociedad.

Palabras clave: tributación, cultura tributaria, política fiscal, sistema tributario

\begin{abstract}
Fiscal policy and its impact on the tax culture of Ecuador is a documentary-type investigation that made it possible to determine that the administration of fiscal policy is oriented towards the generation of income that is destined to satisfy the needs of society; but that this is affected by tax evasion; which is one of the greatest phenomena that directly affects the tax system of Ecuador. Intentional failure to pay contributions not only erodes state income, but deteriorates the structure and economy of a country, causing a damaging effect on society.
\end{abstract}

key words: taxation, tax culture, tax policy, tax system.

\section{Introducción}

La política fiscal es una rama de la política económica, su administración está encaminada a la generación de ingresos que permitan establecer la atención de necesidades de la sociedad, su principal función es la de impulsar

\footnotetext{
${ }^{1}$ Docente Investigador. Carrera de contabilidad. Instituto Superior Tecnológico Daniel Álvarez Burneo. Email: snsiguenza@itsmaristaloja.edu.ec

2 Docente Investigador. Carrera de contabilidad. Instituto Superior Tecnológico Daniel Álvarez Burneo. Email: kssandoval@itsmaristaloja.edu.ec

${ }^{3}$ Docente Investigador. Carrera de contabilidad. Instituto Superior Tecnológico Daniel Álvarez Burneo. Email: amvizcaino@itsmaristaloja.edu.ec

${ }^{4}$ Docente Investigador. Carrera de contabilidad. Instituto Superior Tecnológico Daniel Álvarez Burneo. Email: eagiron@itsmaristaloja.edu.ec

${ }^{5}$ Docente Investigador. Carrera de contabilidad. Instituto Superior Tecnológico Daniel Álvarez Burneo. Email: sperazo@itsmaristaloja.edu.ec

${ }^{6}$ Docente Investigador. Carrera de contabilidad. Instituto Superior Tecnológico Daniel Álvarez Burneo. Email: lejimbo@itsmaristaloja.edu.ec
} 
la estabilidad económica mediante el control de los dos principales instrumentos de la política fiscal: el gasto público y los impuestos. y de esta forma contribuir a enfrentar la desigualdad (Gamboa, Hurtado, \& Ortiz, 2017)

Para comprender cómo la política fiscal influye en el crecimiento económico hay que analizar la incidencia de los impuestos, regulaciones y gasto público en cada una de sus fuentes de financiamiento para que el Estado cumpla con su rol y la distribución de tales recursos. El financiamiento puede provenir de diferentes fuentes o tributos: impuestos, tasas, contribuciones especiales, ingresos patrimoniales (provenientes de la explotación de recursos naturales de propiedad estatal), transferencias y donaciones ya sean internas o externas y, endeudamiento. De los recursos referidos, los tributos y específicamente los impuestos constituyen el instrumento más recomendado para sostener las finanzas públicas, al ser entradas permanentes permiten establecer una planificación a largo plazo para la construcción de una sociedad más equitativa y justa (Córdova, 2017).

La política fiscal está relacionada con los ingresos que recauda un gobierno para poder suplir las necesidades de la sociedad. Los ingresos se obtienen de la recaudación de tributos como el IVA y el Impuesto a la Renta, también por la venta de bienes o servicios; transferencias y donaciones que se reciben, entre otros; con la finalidad de poder mantener la estabilidad económica de un país.

Algunos países cuentan con políticas fiscales que operan sobre bases institucionales simples y en otros países dichas bases son considerablemente más avanzadas (Arenas de Mesa, 2016). Igualmente,

Hay casos en que las autoridades fiscales gozan de credibilidad y cuentan con la confianza de la ciudadanía, mientras que en otros su rango de acción es menor, debido principalmente a la falta de confianza y a la debilidad institucional. Cuando la política fiscal avanza hacia la sostenibilidad fiscal, se fortalecen los dos pilares del desarrollo sostenido (crecimiento inclusivo e igualdad) (Arena, 2016, p. 28).

La sostenibilidad fiscal está muy ligada al crecimiento económico, la productividad y al fortalecimiento de los ingresos públicos a través de mayores bases y cargas tributarias. Para lograrla se debe mejorar la progresividad de la estructura tributaria, fortalecer los impuestos a la renta personal y a la propiedad, reducir la evasión fiscal (alcanzó alrededor del 6,3\% del PIB en 2017), reevaluando los gastos tributarios (el 3,7\% del PIB) y avanzando en una nueva generación de impuestos relacionados con la economía digital, el medioambiente y la salud pública. La mejora de la calidad, la eficiencia y la eficacia del gasto es también central para la sostenibilidad (Comisión Económica para América Latina y el Caribe, CEPAL, 2019).

La sostenibilidad fiscal es esencial para entregar estabilidad financiera a las distintas áreas de las políticas públicas, es por ello que, el desafío de la política fiscal y de la sostenibilidad fiscal será "avanzar en la construcción de un marco analítico y de bases sólidas para lograr, entre otros, el objetivo de dar gobernabilidad al crecimiento en América Latina. Sin sostenibilidad fiscal no será posible avanzar en un desarrollo sostenido" (Arenas de Mesa, 2016, p. 21).

\subsection{La política fiscal en América Latina}

América Latina en materia fiscal ha presenciado importantes cambios a pesar de la heterogeneidad de los países que la componen.

Entre 2000 y 2011 los ingresos fiscales totales de América Latina aumentaron del 19,6\% al 23,6\% del PIB y del $24,5 \%$ al $28,3 \%$ en el Caribe, mientras que la carga tributaria (excluidos los aportes a la seguridad social) aumentó del $12,7 \%$ al $15,7 \%$ en América Latina y del $19,3 \%$ al $23,0 \%$ del PIB en el Caribe. Los mayores incrementos de la carga tributaria (en el sentido restringido, sin seguridad social) se observaron en la Argentina y el Ecuador y alcanzaron dos o más puntos porcentuales del PIB en la mayor parte de países (CEPAL, 2013, p. 5). 
En el Ecuador, gracias a la mejora en el precio internacional de petróleo crudo, aumentaron los ingresos petroleros por exportaciones: en 2018 ascendieron al 2,1\% del PIB, frente a un 1,6\% en 2017. En El Salvador, el aumento de los ingresos totales se debe a la evolución de los ingresos tributarios, que fueron impulsados por un alza en la recaudación de los pagos del IVA asociado con las importaciones (CEPAL, 2019, p. 30).

En cuanto a los ingresos públicos de América Latina, estos se mantuvieron por dos años seguido (2017 y 2018) en el mismo nivel, 18,1\% del PIB, según la CEPAL (2019):

Esta estabilidad ocultó tendencias opuestas entre los grupos de países: se produjo una tendencia a la reducción en el grupo de países compuesto por Centroamérica y México, mientras que en América del Sur estos ingresos están adquiriendo mayor dinamismo, en el Caribe, hubo un incremento de la recaudación tributaria y de ingresos por otros conceptos, incluidos los provenientes de programas de ciudanía por inversión, impulsó la evolución de los ingresos totales, que alcanzaron un 27,6\% del PIB en 2018, frente a un 26,3\% del PIB en 2017. (p. 7)

Para, Barreix \& Corrales (2019) los ingresos públicos han estado aumentando sostenidamente en los últimos años, debido a un crecimiento de los ingresos tributarios. Los sistemas tributarios de la región se caracterizan por una alta participación de los impuestos indirectos (impuestos al consumo, como el IVA), por encima de los impuestos directos (como el impuesto a la renta), debido a que, entre otros factores, la recaudación de los primeros es mucho más sencilla.

En cuanto al gasto público en América Latina, puede considerarse procíclico, característica usual en países en desarrollo y según Barreix \& Corrales (2019) exponen que, "la composición del gasto tiene también un sesgo contra la inversión pública que ha perdido terreno frente al gasto corriente, tanto como porcentaje del gasto total como en términos per cápita". (p. 6)

El déficit primario en el conjunto de los países de América Latina se redujo del 0,8\% del PIB en 2017 al $0,5 \%$ del PIB en 2018. Este ajuste se debió principalmente al recorte registrado en el gasto primario los gastos totales excluidos los pagos de intereses-, que disminuyó de un 19,0\% del PIB en 2017 a un 18,6\% del PIB en 2018. En el Caribe, el superávit primario aumentó del 0,9\% al 2,1\% del PIB en el mismo período, lo que se condice con la necesidad de continuar generando superávits primarios sustanciales para estabilizar y reducir el elevado nivel de deuda pública (CEPAL, 2019, p. 9).

En lo referente a la deuda pública bruta, la CEPAL (2019) expone que esta tiende al alza y que alcanzó un $42,3 \%$ del PIB en el año 2018, frente al 39,4\% del PIB registrado en el año 2017, resultados que se debe porque la deuda bruta de Argentina aumentó 38 puntos porcentuales del PIB entre 2017 y el tercer trimestre de 2018, alcanzando un nivel del $95,4 \%$ del PIB.

\subsection{El sistema tributario}

El sistema tributario representa un instrumento de la política económica que permite a la política fiscal la dotación de ingresos permanentes al Estado y de esta manera pueda cumplir con sus funciones, fomentando la producción y la generación de empleo; el sistema tributario es un factor indispensable para el funcionamiento, financiamiento y crecimiento de una economía, donde el método de recaudo depende de la política fiscal vigente (Ruiz , Arias, Ibarra, \& Sanandrés, 2018).

Para su aplicación según, Washco (2015) se deben considerar los siguientes elementos en su estructura: 
Figura 1

Elementos del Sistema Tributario

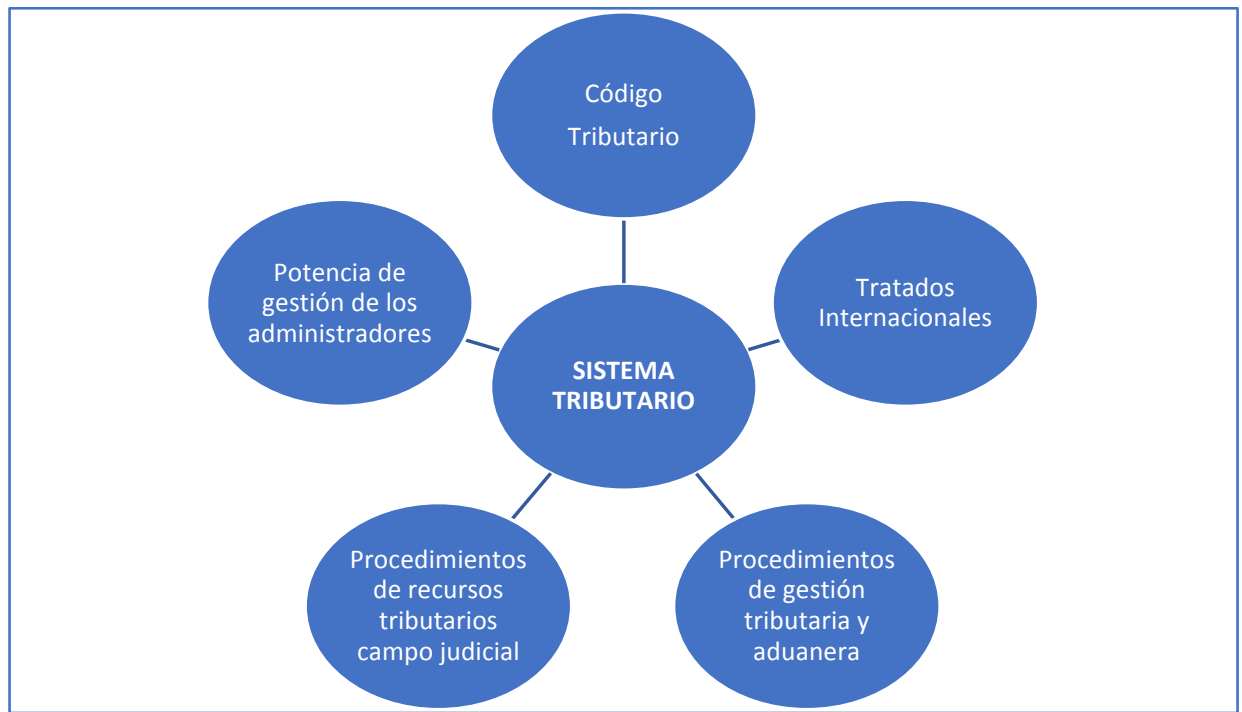

Fuente: (Washco, 2015)

El sistema tributario se traduce en un conjunto de tributos que rigen en un determinado tiempo y espacio, y que están relacionados con la normativa constitucional e íntimamente vinculado con la política económica, cuyo fin se enfoca en la minimización de costos en la recaudación y cumplimiento de la política fiscal (Ruiz et al., 2018).

Los tributos se conciben como la manera de obtener ingresos que los gobiernos utilizan para cubrir gastos públicos, estos vienen a través de impuestos, tasas y contribuciones especiales o de mejora, que conforma el sistema tributario. De esta manera, la estructura tributaria se encuentra relacionada con los Impuestos tales como: el impuesto a la renta, impuesto al valor agregado, impuesto al consumo, entre otros, con particularidades de cada país (Quispe, Arellano, Rodríguez, Negrete, \& Vélez, 2019).

En Ecuador, el sistema tributario según Garzón, Ahmed, \& Peñaherrera (2018), lo constituyen un conjunto de impuestos que la Ley exige su cumplimiento, los mismos que son administrados por el Servicio de Rentas Internas (SRI), cumpliendo el objetivo de estimular el ahorro, fomentar la inversión y el empleo; así como lograr una distribución equitativa de la riqueza (Pallo, 2015).

De acuerdo a lo estipulado en el Código Tributario (2016), en el Ecuador, el sujeto activo del tributo es el Estado, representado por el SRI, organismo encargado de la recaudación y control; y, el contribuyente, como el sujeto pasivo de la obligación tributaria. En tal sentido, Baistrocchi (2017) considera que los ingresos tributarios recaudados por el SRI tienen como destino principal, financiar el Presupuesto General del Estado; para ejemplificar, según el Ministerio de Finanzas en el año 2016, los ingresos fiscales ascendieron a 14 989,70 millones de dólares, equivalente al 50,24 \% de dicho presupuesto, convirtiéndose en una fuente importante de ingresos para el gobierno central (Garzón, Ahmed, \& Peñaherrera, 2018, p. 41).

El SRI nació el 2 de diciembre de 1997, basándose en los principios de justicia y equidad, como respuesta a la alta evasión tributaria. iHacerle bien al país!, es el slogan que identifica a la institución y que ha sido desde el 2007 el estandarte de la lucha continua contra la evasión y las malas prácticas tributarias, y de la búsqueda de la mejora continua en beneficio de los contribuyentes y del país.

La labor efectuada por el SRI durante los últimos años se evidencia en un enorme incremento en la recaudación de impuestos, según lo expuesto en su página web: 
Entre los años 2000 y 2006 la recaudación fue de 21.995 millones; mientras que en el período comprendido entre 2007 y 2013 la recaudación se triplicó, superando 60.000 millones de dólares. La cifra alcanzada por el SRI no se debe a reformas tributarias sino a la eficiencia en la gestión de la institución, a las mejoras e implementación de sistemas de alta tecnología, desarrollo de productos innovadores como la Facturación Electrónica, SRI móvil, servicios en línea, reducción de costos indirectos a la ciudadanía y el afianzamiento de la cultura tributaria, además del incremento significativo de contribuyentes.

De esta manera es importante que la recaudación tributaria genere en la economía un sustento financiero que atienda las necesidades del entorno (Gamboa, Hurtado, \& Ortiz, 2017).

\subsubsection{Régimen tributario}

Los regímenes tributarios se definen como las categorías bajo las cuales una persona natural o jurídica que posee o va a iniciar un negocio debe estar registrada en los registros de la autoridad tributaria de un Estado en particular (plataforma digital única del estado Peruano, 2019); en Ecuador además de considerar el régimen general o régimen único de contribuyentes $(R U C)$ se ha establecido un régimen simplificado para el pago de los impuestos; el régimen impositivo simplificado ecuatoriano (RISE), el cual brinda facilidades para que los pequeños contribuyentes cumplan con sus obligaciones de manera sencilla a través de la cancelación de cuotas mensuales o anuales que los exoneran de la declaración del impuesto al valor agregado (IVA) y del impuesto a la renta (IR), además de facilitar la entrega de documentos de venta a través de la emisión de Notas de Venta Simplificadas (Ruso, Villamar , Ordeñana, \& Contreras, 2019).

Se considera que el régimen tributario ecuatoriano debe estar constituido por las normas que regulen el vínculo jurídico que existe entre los sujetos pasivos (contribuyentes) y el sujeto activo (estado) para el pago de los tributos; tomando en cuenta que, para su aplicación, el mismo deberá regirse por los principios de legalidad, generalidad, igualdad, proporcionalidad e irretroactividad de acuerdo como lo manifiesta el Código Tributario (2018) en el artículo 5.

\subsubsection{La cultura tributaria y sus conflictos}

La cultura tributaria se debe entender como un conjunto de valores, conocimientos y actitudes compartidos por los miembros de una sociedad respecto a la tributación y la observancia de las leyes que la rigen, que conducen al cumplimiento permanente y voluntario de los deberes tributarios con base en la razón, la confianza y la afirmación de los valores de ética personal, respeto a la ley, responsabilidad ciudadana y solidaridad social, tanto de los contribuyentes, como de los funcionarios de las diferentes administraciones tributarias (Díaz, Cruz, \& Castillo, 2016, p. 700).

Bonilla (2014) define a la cultura tributaria como el conjunto de acciones, que buscan conseguir que la ciudadanía se convenza de la posibilidad, la necesidad y la conveniencia de una fiscalidad justa. La cultura tributaria asume el compromiso de educar a la ciudadanía en temas fiscales, que promuevan el cumplimiento de las responsabilidades del contribuyente, consciente del buen uso de los bienes públicos.

Para, Gómez, Jiménez, \& Martner (2017) es importante priorizar "una cultura tributaria en la que se penalice efectivamente a los evasores y, además, se entienda que los ingresos tributarios constituyen la base fundamental de financiamiento de las funciones esenciales de un Estado moderno" (p. 361), con la finalidad de contrarrestar la evasión fiscal.

La evasión fiscal es uno de los mayores fenómenos que afectan directamente el sistema tributario de un país, el no pago intencional de las contribuciones no sólo erosiona los ingresos del Estado, sino que deteriora la estructura y economía de un país, causando un efecto dañino para la sociedad (Morales, Ruiz , \& Ycaza, 2009). 
En cuanta a la problemática de la evasión fiscal la CEPAL expone en su publicación titulada: Panorama Fiscal de América Latina y el Caribe (2019), lo siguiente:

Una de las principales barreras para la movilización de recursos internos es el alto nivel de evasión fiscal y los flujos financieros ilícitos. La última estimación de la CEPAL sugiere que el costo de la evasión y la elusión fiscal en América Latina alcanzó un 6,3\% del PIB en 2017, cifra equivalente a 335.000 millones de dólares. La CEPAL estima también que los flujos financieros ilícitos que salen de la región como consecuencia de la manipulación de precios del comercio internacional de bienes alcanzaron los 85.000 millones de dólares en 2016, monto equivalente al 1,5\% del PIB de América Latina y el Caribe. (p. 7)

Ademas, la CEPAL (2019) manifiesta que, "el principal desafío de la política económica es evitar que la región se estanque en el ámbito económico y social, así como preservar los avances en materia de estabilidad macrofinanciera y sostenibilidad de la deuda" (p. 11). También definen que, el papel de la política fiscal debe desarrollarse en tres funciones: estabilización, provisión de bienes públicos y redistribución.

Ante lo expuesto, Gómez \& Morán (2016) mencionan que, es indispensable que se priorice la construcción de una cultura tributaria en la que se sancione a los contribuyentes evasores y que se comprenda que los ingresos tributarios son la base para el financiamiento del presupuesto general de un Estado. Lo cual requiere transparencia en la gestión de la administración tributaria y de las demás instituciones que intervienen en las finanzas de un país, para así garantizar un desarrollo económico participativo y sostenible.

El presente trabajo pretende responder a la pregunta de investigación ¿Cuál es la incidencia en la aplicación de la Política Fiscal frente a la cultura tributaria en Ecuador?, debido a que, los contribuyentes prefieren convertirse en evasores del impuesto, ya sea por el simple desconocimiento de la norma o porque les genera desconfianza el destino que da el Estado a los dineros que se recaudan (Fernandez, Herrera, Tamayo, Rojas, \& Martínez, 2018).

\section{Metodología}

El diseño de la investigación obedece al enfoque cualitativo, cuya principal característica es la exploración de los fenómenos en profundidad, buscando los significados en los datos, manteniendo una mente abierta (Hernández, Fernández, \& Baptista, 2010).

Para efectuar un análisis de la literatura existente y las fuentes de investigación referente a la política fiscal y su incidencia en la cultura tributaria del Ecuador, se decide realizar, una investigación de tipo documental, la cual constituye un procedimiento científico y sistemático de indagación, recolección, organización, análisis e interpretación de información o datos en torno a un determinado tema. Utilizando un estudio de mapeo sistemático de literatura (Systematic Mapping Study, SMS) según las orientaciones de Petersen, Feldt, Mujtaba, \& Mattsson (2008), se realizó la clasificación y análisis de temáticas para obtener un mapa visual del conocimiento existente dentro del campo de la política fiscal y la cultura tributaria en el Ecuador.

En la figura 2 se esquematiza el proceso metodológico para la identificación de información y estudios primarios en base al mapeo sistemático. 
Figura 2

Proceso del Mapeo Sistemático (SMS)

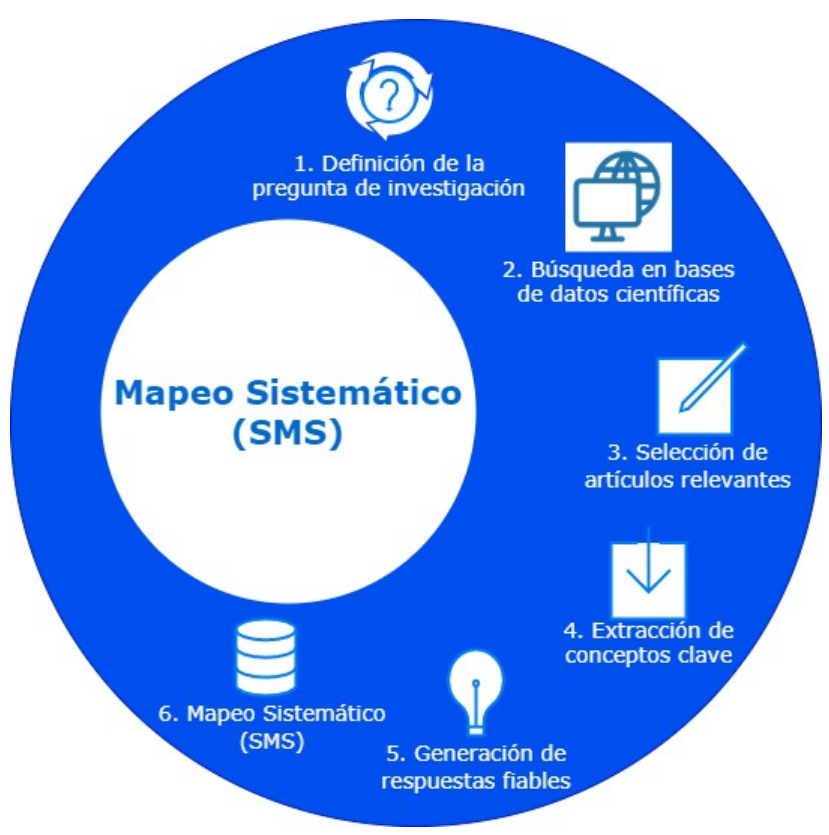

Fuente: (Petersen, Feldt, Mujtaba, \& Mattsson, 2008)

Por lo tanto, para llevar a cabo el mapeo sistemático es necesario aplicar el método de revisión bibliográfica, que consiste en la selección de las fuentes de información, tanto directa como indirecta: libros, documentos e informes normativos de instituciones profesionales y gubernamentales; ponencias y artículos científicos de bases de datos electrónicas, con vistas a extraer las informaciones e ideas más relevantes, relacionadas con el objeto de investigación.

En este sentido, se consideran los planteamientos de Hoyos (2010), quien establece las fases que se deben desarrollar dentro de una investigación documental, ahondando en tres fases que son: la preparatoria, descriptiva e interpretativa.

\subsection{Fase preparatoria}

Para el desarrollo de esta fase se plantean cinco criterios que permiten situar la investigación en una ruta confiable para la selección del material objeto de estudio. Estos criterios se detallan a continuación:

- Acceso a información en bases de datos. En el proceso de obtención de información de relevancia científica, se identifica nueve bases de datos electrónicas utilizadas en diferentes ramas de la ciencia en general; lo cual se evidencia en la tabla 1. 
Tabla 1: Bases de datos electrónicas consultadas

\begin{tabular}{cll}
\hline Id & \multicolumn{1}{c}{ Base de Datos } & \multicolumn{1}{c}{ Enlace } \\
\hline 1 & Dialnet & www.dialnet.unirioja.es \\
2 & Elsevier & www.elsevier.com \\
3 & Espacios & www.revistaespacios.com \\
4 & Redalyc & www.redalyc.org \\
5 & ResearchGate & www.researchgate.net \\
6 & Scielo & www.scielo.org \\
7 & ScienceDirect & www.sciencedirect.com \\
8 & Scopus & www.scopus.com \\
9 & Taylor \& Francis & taylorandfrancis.com \\
\hline
\end{tabular}

Elaborado por: Las autoras

- Tipología de bases de datos, teniendo en cuenta la identificación de la publicación (ISSN) y del libro (ISBN).

- Selección de artículos relacionados con el tema objeto de estudio, utilizando una estrategia de búsqueda formal considerando el título y palabras clave en bases de datos electrónicas y revistas indexadas.

- Tiempo de Publicación de los artículos (últimos 10 años).

\subsection{Fase Descriptiva}

Para la definición de las unidades de análisis se parte de grupos de palabras establecidas por similitud semántica y conceptual, relevante y significativa en el estudio de la política fiscal y la cultura tributaria. Entre tanto, se indaga la literatura y se opta por factores destacados, resaltando palabras en el título, resumen y palabras claves de los artículos seleccionados.

Según Quezada, Garbajosa, Enciso, Solano, \& Barba (2015), once criterios relacionados con la calidad, se toman en cuenta al evaluar los estudios identificados en el mapeo sistemático, los cuales se detallan en la tabla 2.

Tabla 2: Criterios de Calidad

1. ¿Está el papel basado en la investigación (o es simplemente un informe "lecciones aprendidas", basada en la opinión de expertos)?

2. ¿Existe una declaración clara de los objetivos de la investigación?

3. ¿Existe una descripción adecuada del contexto en el que se llevó a cabo la investigación?

4. ¿ ¿Fue el diseño de investigación apropiado para abordar los objetivos de la investigación?

5. ¿¿Fue la estrategia de contratación adecuada a los objetivos de la investigación?

6. ¿Hubo un grupo de control con el cual comparar los tratamientos?

7. ¿Fue de los datos recogidos de una manera que abordó el tema de investigación?

8. ¿Fue el análisis de datos suficientemente rigurosos?

9. ¿A la relación entre el investigador y los participantes han considerado a un nivel adecuado?

10. ¿Existe una declaración clara de los resultados?

11. ¿Es el estudio de valor para la investigación o en la práctica?

Fuente: (Quezada, Garbajosa, Enciso, Solano, \& Barba, 2015) 


\subsection{Fase Interpretativa}

Dentro de la tercera fase denominada interpretativa, se lleva a cabo la revisión minuciosa de la literatura contenida en el material objeto de estudio, para luego realizar una selección y valoración de las teorías que sustentarán los resultados de la investigación realizada.

Para lo cual se elabora una tabla de análisis y síntesis, en la cual se incluye información esencial procedente de cada estudio de acuerdo al objetivo planteado en la investigación, para poder realizar una comparación. Esta tabla permite identificar los hallazgos que son comunes en los diferentes estudios y comparar los resultados de varios estudios.

\section{Resultados}

El análisis de contenidos de los documentos relacionados con el tema la política fiscal y su incidencia en la cultura tributaria del Ecuador obtenidos de la base de datos de Dialnet, Elsevier, Espacios, Redalyc, ResearchGate, Scielo, ScienceDirect y Scopus, reveló la existencia de la siguiente información.

\subsection{La política fiscal}

En la investigación titulada, Gestión de la política fiscal para fortalecer la cultura tributaria en Ecuador, de Gamboa, Hurtado, \& Ortiz (2017), define a la política fiscal como:

La generadora de instrumentos legales, que permitan una efectiva recaudación tributaria, la cual se refleje en el cumplimiento de los objetivos y a la vez permite configurar mediante las recaudaciones tributarias el presupuesto estatal, mismo que está destinado a asegurar la estabilidad económica del país y entonces mejorara el crecimiento de recursos productivos de la sociedad. (p. 460)

En la investigación titulada, Sostenibilidad fiscal y reformas tributarias en América Latina, de Arenas de Mesa, 2016), define a la administración tributaria como:

La encargada de la recaudación de los ingresos públicos (por medio de instituciones de recaudación tributaria y, en algunos casos, empresas del Estado); por otro, una institucionalidad presupuestaria encargada de gestionar, aprobar, ejecutar y evaluar el gasto público, y -por último- la gestión financiera de la deuda pública. Su evolución ha dado origen a leyes de responsabilidad y transparencia fiscal, reglas fiscales y la creación de fondos de estabilización, que han contribuido a la construcción de una arquitectura fiscal contracíclica en la región. (pp. 29, 30)

Es decir, que la administración de la política fiscal está enfocada en la generación de ingresos que permiten satisfacer las necesidades básicas de la sociedad; la política fiscal es uno de los parámetros que el Estado puede controlar para moderar las fluctuaciones y estabilizar la economía a través de los pactos fiscales y los acuerdos tributarios transversales, que son una pieza fundamental para el desarrollo e implementación de políticas públicas que promuevan el crecimiento y enfrenten la desigualdad (Arenas de Mesa, 2016, p. 22).

En relación a Ecuador, en la investigación de Arévalo (2014), titulada, Ecuador: economía y política de la revolución ciudadana, evaluación preliminar, manifiesta lo siguiente:

En Ecuador, los ingresos fiscales dependen en su mayor parte de la volatilidad de los precios del petróleo en el mercado internacional. El petróleo constituye el primer recurso de exportación y la fuente primaria y esencial de los ingresos tributarios. Por lo mismo y en razón de la vulnerabilidad del ingreso y gasto estatal, el Gobierno buscó mejorar los ingresos de la nación mediante reformas tributarias que redujeron la evasión y elusión y permitieron el aumento del recaudo, objetivo que se viene cumpliendo durante la vigencia del actual mandato presidencial. (p. 124, 125) 
Además, los resultados fiscales del Ecuador "provienen es su mayor parte del impuesto al consumo, impuesto al valor agregado IVA, e impuesto al ingreso y en menor medida de las contribuciones a la seguridad social y otros impuestos derivados como los aranceles al comercio exterior" (Arévalo, 2014, p. 128). En este contexto, Quispe et al. (2017) exponen que, "en la República del Ecuador la estructura tributaria está compuesto por: impuestos directos, impuestos indirectos, impuestos gubernamentales, impuestos municipales, seguridad social y otras contribuciones a instituciones y organismos de control". (p. 4)

En tal sentido, "la recaudación tributaria contribuye a los ingresos gubernamentales, constituyéndose una herramienta esencial para el desarrollo de las economías en los países de América Latina" (Quispe et al., 2017, p. 2); y de acuerdo a Bedoya \& Vásconez (2010):

La Administración Tributaria debe enfocar sus esfuerzos en dos grandes líneas de acción. Primero, mejorar la selección de contribuyentes sujetos a control en el marco de una adecuada segmentación que logre influenciar a que los 'contribuyentes oportunistas' tengan suficientes incentivos para que sean 'leales'. Segundo, fortalecer los servicios al contribuyente para reducir los costos de cumplimiento al mínimo y, al mismo tiempo, ampliar y especializar la capacitación que requiere la sociedad. (p. 125)

\subsection{La evasión un factor que interfiere en el cumplimiento de la política fiscal}

En cuanto a esta problemática, Onofre, Aguirre, \& Murillo (2017), en su investigación titulada: La cultura tributaria y su incidencia en la recaudación de los tributos en el Cantón Babahoyo, Provincia de los Ríos, exponen:

La lucha contra la evasión tributaria y el contrabando constituye hoy en día un tema central en todas las agendas políticas de un país, debido a su elevado impacto en la estabilidad económica, se hace necesario concienciar a la población a través de una cultura tributaria, en las estrategias de desarrollo y en la gobernabilidad en general.

Aunque las estrategias de control o fiscalización tributaria son fundamentales en dicha lucha, resulta claro que el esfuerzo por controlar el cumplimiento tributario y generar un riesgo creíble ante el incumplimiento no basta por sí solo para vencer las prácticas de evasión. (p. 50)

Torres (2012) definen a la evasión tributaria como una falta en el cumplimiento de las obligaciones de carácter tributario por parte de los contribuyentes sean estas personas naturales o jurídicas. Osorio (2016) pone de manifiesto que esta práctica se ha convertido en uno de los principales puntos débiles que presenta la economía en toda América Latina.

Si bien es cierto, hay factores que pueden afectar la relación costo-beneficio de la evasión, como la probabilidad de ser auditado y los castigos monetarios y judiciales a los que se expone un evasor, existen otras variables políticas, sociales y culturales que inciden en la decisión del contribuyente. Por ejemplo, su percepción acerca del grado de justicia existente en la relación Estado-sociedad y del funcionamiento de las instituciones.

En tal sentido, no son sorprendentes los hallazgos que exponen autores como Bergman (2009), que indica que los individuos que son auditados y eventualmente castigados por evadir impuestos no demuestran una mayor probabilidad de cumplir con sus obligaciones fiscales a lo posterior, lo que hace difícil que un ciudadano cumpla de manera efectiva con el pago de sus obligaciones tributarias si en su sociedad la evasión se ha convertido en una práctica común.

En el análisis realizado por Quispe et al. (2017), en su artículo titulado: Las reformas tributarias en el Ecuador. Análisis del periodo 1492 a 2015, exponen que, en nuestro país (Ecuador) también se evidencia una alta concentración de impuestos a los consumos, evasión y elusión tributaria, una débil participación en la recaudación de los impuestos directos, elementos que han incidido para que los gobiernos de turno busquen la aplicación de nuevas políticas tributarias que optimicen la recaudación de los tributos. 
La causa de la evasión tributaria en Ecuador, según Morales, Ruiz, \& Ycaza (2009), tiene que ver con el comportamiento de los contribuyentes, su moral, la percepción que tienen acerca de la equidad del sistema y el trato que reciben de la administración tributaria. Por lo que, un mayor nivel de conciencia cívica respecto al cumplimiento tributario, junto a una percepción de riesgo efectivo por el incumplimiento, permitirá disminuir en Ecuador los elevados índices de evasión y contrabando existentes, siendo necesario e imprescindible desarrollar una cultura tributaria, que permita a la ciudadanía admitir las obligaciones tributarias como un deber sustantivo, acorde con los valores democráticos (Onofre, Aguirre, \& Murillo, 2017).

Según, Marandu, Mbekomize, \& Ifezue (2015), para inducir a que los contribuyentes paguen sus impuestos, sería importante que los encargados de la política fiscal de un país, utilicen métodos que generen un cambio de normas y por ende de consciencia y moral tributaria; así como métodos que impliquen sanciones a través de un control subjetivo.

\subsection{La moral tributaria}

La moral tributaria como factor que incide en el cumplimiento de la política fiscal y que se entiende como la disposición personal de los contribuyentes para pagar los impuestos (Torgler \& Schaltegger, 2005); ha sido objeto de una serie de estudios, destacando que sus mayores investigaciones se ubican en la primera década del siglo $\mathrm{XXI}$, como respuesta a las falencias de los modelos establecidos en los años setenta, para explicar la evasión; desde un enfoque económico, la misma moral tributaria ha evolucionado de manera notable, incorporando nuevos elementos en su estudio, tales como la edad de los individuos, su nivel educativo y la percepción de este frente a las acciones de tipo gubernamental (Castañeda, 2015).

Desde la perspectiva de la administración tributaria, la moral tributaria según Lozano \& Tamayo (2016), "resulta indispensable gestionar la ética como mecanismo para prevenir la corrupción, para lo cual la elaboración consensuada de un código de ética resulta fundamental, así como su implementación, seguimiento y debida mitigación de riesgos". (p. 31). Esto permitiría que la administración tributaria ofreciera ética y transparencia a los contribuyentes a cambio de cumplimiento tributario. A largo plazo se construye una relación ética y recíproca entre contribuyentes y la administración, quien será la encargada de liderar el sostenimiento de un trato adecuado entre las partes (Lozano \& Tamayo, 2016, p. 31).

La moral fiscal, motiva al contribuyente a pagar sus impuestos, pero teniendo en cuenta que, al mismo tiempo sus cálculos racionales acerca de las oportunidades reales que tiene de defraudar a Hacienda. Consecuentemente, no todos los individuos evadirían los impuestos: solo lo harían aquellos que carecen de moral fiscal y en la medida en que consideren que tienen una oportunidad, es decir, que crean tener una probabilidad muy baja de ser descubiertos y/o sancionados (Giachi, 2014, p.78).

En tal sentido, Castañeda (2015) en su trabajo de investigación titulado: La moral tributaria en América Latina y la corrupción como uno de sus determinantes, concluye que:

El tema de la moral tributaria es central en lo que atañe al estudio de la capacidad fiscal de un Estado, aunque el análisis normativo de la tributación lo desconoce al asumir que los contribuyentes aceptan lo que por ley les corresponde pagar (análisis de la tributación óptima), a la vez que consideran racionalmente los costos y beneficios de evadir. No obstante, el grado en que un ciudadano asume su responsabilidad fiscal no está mediada sólo por consideraciones económicas, dado que también son importantes aspectos como la evaluación que hace de la relación Estado-sociedad, con base en su experiencia y en referentes construidos socialmente. (p.125)

Para fomentar la moral tributaria en los contribuyentes, Bedoya \& Vásconez (2010) declaran en su investigación titulada: Entendiendo la moral Tributaria en Ecuador, lo siguiente: 
Dentro de la conceptualización de Moral del Estado, el conocimiento del destino del gasto es fundamental, por lo cual una adecuada publicidad de la gestión del gasto público influencia positivamente en la Moral del Contribuyente, dados los resultados obtenidos. Podemos ampliar el concepto Transparencia y Publicidad en lo que en términos anglosajones se denomina Accountability, que implica la obligación de reportar, explicar o justificar las acciones de los funcionarios públicos a la sociedad. En este sentido, los ciudadanos que perciben positivamente el destino de su contribución tienen una mayor probabilidad de pagar sus impuestos, como un acto de corresponsabilidad. (p. 30)

\subsection{La cultura tributaria}

Gómez \& Morán (2016) mencionan que, es indispensable que se priorice la construcción de una cultura tributaria en la que se sancione a los contribuyentes evasores y que se comprenda que los ingresos tributarios son la base para el financiamiento del presupuesto general de un Estado. Lo cual requiere transparencia en la gestión de la administración tributaria y de las demás instituciones que intervienen en las finanzas del país, para así garantizar un desarrollo económico participativo y sostenible.

Por ende, sería importante considerar la alternativa de construir un índice de cultura fiscal en base a la aceptación que presentan los contribuyentes, referente a la gestión del régimen tributario de cada país, para posteriormente efectuar un análisis comparativo con otros datos relacionados a diversos contextos políticos y económicos de la región (Giachi, 2014).

Finalmente, Marandu, Mbekomize, \& Ifezue (2015), en su artículo titulado: Determinants of Tax Compliance: A Review of Factors and Conceptualizations, realizan una búsqueda de literatura en la base de datos de publicaciones de la Universidad de Botswana para estudios que giraron entre el período de 1985 y 2012; en donde concluyen que:

El gobierno puede desarrollar un programa educativo que tenga tres temas con el objetivo de construir una cultura de cumplimiento tributario. El primer tema puede ser crear la percepción de que los ingresos fiscales se están utilizando correctamente (actitud). El segundo tema puede ser un programa en los medios de comunicación que muestre ejemplos de empresarios que pagan impuestos y cómo estos impuestos se han utilizado para mejorar el desarrollo del país y crear empleo. (pp. 216-217)

\section{Conclusiones}

La política económica de un país se maneja a través de un Presupuesto general del Estado (PGE), el cual siempre se han configurado mediante los ingresos y gastos públicos, por lo tanto a nivel de Latinoamérica la mayor fuente de ingresos luego del petróleo, son los tributos constituyéndose en impuestos, tasas y contribuciones; de aquí la magnitud de que una adecuada política fiscal en un gobierno, gestione los ingresos necesarios que permitan financiar obras de interés social tanto en educación, salud, vialidad e infraestructura; por ende, los impuestos no sólo tienen un fin económico sino también un fin social en dónde la moral y cultura tributaria de todos los contribuyentes es la columna vertebral para que un régimen tributario funcione en base a una eficiente redistribución de la riqueza por parte de la administración pública.

Según la Organización para la Cooperación y Desarrollo Económico (OCDE, 2016), en los últimos 25 años los ingresos fiscales correspondientes a fuentes tributarias y de seguridad social han aumentado considerablemente en América Latina, es por ello que una política fiscal estratégicamente estructurada, es un avance crucial en la economía de un país, por ende el gran desafío de las administraciones tributarias y en especial de la ecuatoriana es concientizar a la ciudadanía de que el pago de sus impuestos afecta de manera directa a la distribución del gasto público; y de esa forma evitar situaciones adversas como evasión, elusión o fraude fiscal. 
La recaudación tributaria respecto al PIB en el Ecuador evidenció cambios significativos a partir de 2008; un mecanismo clave fue el cambio en el marco jurídico, puesto que la nueva Constitución hace explícito que el régimen tributario se regirá por los principios de generalidad, progresividad, eficiencia, simplicidad administrativa, irretroactividad, equidad, transparencia y suficiencia recaudatoria; constituyendo estos principios la base del sistema tributario en el Ecuador, y el pilar fundamental para el establecimiento de leyes y lineamientos para el funcionamiento del sistema, por tal razón se puede señalar que el régimen tributario nace a partir de los principios tributarios; lo que da vida a la política fiscal de un país.

La educación y cultura tributaria en los contribuyentes en general, todavía es escasa en ciertos sectores de la economía del Ecuador, puesto que el pago voluntario y consciente de los impuestos, aún no es considerada como una responsabilidad preferente en los contribuyentes, por lo que en el país aún existe altos índices de evasión fiscal. Por lo tanto, una de las metas de la administración tributaria, que en el Ecuador es ejercida por el Servicio de Rentas Internas (SRI), es mantener permanentemente un sistema tributario en base a la capacidad contributiva del contribuyente, que lo estimule o motive a pagar voluntariamente sus impuestos bajo un fin no sólo económico sino también social, a través de una óptima distribución del gasto público en dónde ellos visualicen el beneficio obtenido en el cumplimiento de sus obligaciones tributarias, promoviendo de esta manera una enmarcada cultura tributaria a nivel de país.

Finalmente, cabe recalcar que la cultura tributaria no es solamente una actitud de valores o de principios por parte del contribuyente, sino que también implica una labor educativa por parte de la administración tributaria, de manera que a través de la difusión y socialización de la normativa el contribuyente conozca sus responsabilidades como ciudadano fiscal y mantenga una percepción orientada al pago de sus impuestos como una acción de ética social y empresarial.

\section{Referencias bibliográficas}

Arenas de Mesa, A. (2016). Sostenibilidadfiscal y reformas tributarias en América Latina. Santiago: Comisión Económica para América.

Arévalo, G. A. (2014). Ecuador: economía y política de la revolución ciudadana, evaluación preliminar. Apuntes del CENES, 33(58), 109-134.

Barreix, A., \& Corrales, L. F. (2019). Reglas Fiscales Resilientes en América Latina. Washington, D.C.: Banco Interamericano de Desarrollo.

Bedoya, A., \& Vásconez, B. (2010). Entendiendo la moral tributaria en Ecuador. Revista Institucional del Servicio de Rentas Internas(05), 91-132.

Bergman, M. (2009). Tax Evasion and the Rule of Law in Latin America: The Political Culture of Cheating and Compliance in Argentina and Chile. Pennsylvania: The Pennsylvania State University Press.

Bonilla, E. C. (2014). La cultura tributaria como herramienta de política fiscal: la experiencia de Bogotá. Ciudades, estados y política, 1(1), 21-35.

Castañeda, V. M. (2015). La moral tributaria en América Latina y la corrupción como uno de sus determinantes. Revista Mexicana de Ciencias Políticas y Sociales, 60(224), 103-132.

Código Tributario de la República del Ecuador (2018). Obtenido de http://www.ces.gob.ec/lotaip/2018/Agosto/Anexos-literal-a2/CODIGO\%20TRIBUTARIO.pdf

Comisión Económica para América Latina y el Caribe. (2013). Panorama fiscal de América Latina y el Caribe. Reformas tributarias y renovación del pacto fiscal. Santiago: CEPAL. 
Comisión Económica para América Latina y el Caribe. (2019). Panorama Fiscal de América Latina y el Caribe. Santiago: CEPAL.

Córdova, J. N. (2017). La tributación objetiva de pequeños empresarios: una visión comparada entre Ecuador y España, período 2008-2016. (Tesis de Maestría). Instituto Universitario de Investigación en Estudios. Madrid, España.

Díaz, J. C., Cruz, B. G., \& Castillo, N. R. (2016). Cultura Tributaria. Publicando, 3(9), 697-705.

Fernandez, S. R., Herrera, E. J., Tamayo, L. M., Rojas, A., \& Martínez, L. Á. (2018). La evasión de impuesto como variable económica negativa para Colombia. Espacios, 39(50), 2.

Gamboa, J., Hurtado, J., \& Ortiz, G. (2017). Gestión de la política fiscal para fortalecer la cultura tributaria en Ecuador. Publicando, 2(10), 448-461.

Garzón, M. A., Ahmed, A. R., \& Peñaherrera, J. M. (2018). El sistema tributario y su impacto en la Economía Popular y Solidaria en el Ecuador. Revista de Ciencia, Tecnología e Innovación, 5(1), 38-53.

Giachi, S. (2014). Dimensiones sociales del fraude fiscal: confianza y moral fiscal en la España contemporánea. Revista Española de Investigaciones Sociológicas(145), 73-98.

Gómez, J. C., \& Morán, D. (2016). Evasión Tributaria en América Latina. Santiago: CEPAL.

Gómez, J. C., Jiménez, J. P., \& Martner, R. (2017). Consensos y confictos en la política tributaria de América Latina. Santiago: CEPAL.

Hernández, R., Fernández, C., \& Baptista, M. d. (2010). Metodología de la Investigación. México D.F.: McGRAW HILL.

Hoyos, C. (2010). Un modelo para la investigación documental. Guía teórico-práctica sobre construcción de Estados del Arte con importantes reflexiones sobre la investigación. Medellín: Señal Editora.

Lozano, E., \& Tamayo, D. (2016). Gestión de la ética en la administración tributaria colombiana . Revista de Derecho Privado, 2016(55), 1-35.

Marandu, E. E., Mbekomize, C. J., \& Ifezue, A. N. (2015). Determinants of Tax Compliance: A Review of Factors and Conceptualizations. International Journal of Economics and Finance, 7(9), 207-218.

Morales, C., Ruiz , F., \& Ycaza, W. J. (2009). Análisis de la Evasión Fiscal en el Impuesto a la Renta del Ecuador. Obtenido de https://www.dspace.espol.edu.ec/bitstream/123456789/745/1/1396.pdf

Onofre, R. F., Aguirre, C. G., \& Murillo, K. G. (2017). La cultura tributaria y su incidencia en la recaudación de los tributos en el Cantón Babahoyo, Provincia de los Ríos. Dominio de las Ciencias, 3(3), 45-68.

Osorio, L. M. (2016). Reforma tributaria e informalidad laboral en Colombia: Un análisis de equilibrio general dinámico y estocástico. Ensayos sobre Política Económica, 34(80), 126-145.

Pallo, E. L. (2015). Evolución de los Impuestos Directos e Indirectos dentro del Régimen Tributario Ecuatoriano y su Recaudación en el Periodo 2007-2012. (Tesis de Maestría). Universidad de Guayaquil. Guayaquil, Ecuador.

Petersen, K., Feldt, R., Mujtaba, M., \& Mattsson, M. (2008). Systematic Mapping Studies in Software Engineering. Proceedings of the 12yh International Conference on Evaluatión and Assessment in Software Engineering, 17. 
Quezada, P., Garbajosa, J., Enciso, L., Solano, L., \& Barba, L. (2015). Estructura, Componentes e Importancia de los Cuerpos de Conocimiento en el contexto de la Ingeniería de Software y propuesta de dos nuevas áreas de conocimiento en la currícula de la Titulación de Sistemas Informáticos. Revista Tecnológica ESPOL, 28(5), 133-146.

Quispe, G. M., Arellano, O. E., Rodríguez, E. A., Negrete, O. P., \& Vélez, K. G. (2019). Las reformas tributarias en el Ecuador. Análisis del periodo 1492 a 2015. Espacios, 4O(13), 21.

Ruiz , M. V., Arias, I. P., Ibarra, M. D., \& Sanandrés, L. G. (2018). El Sistema Tributario Ecuatoriano y El Presupuesto General del Estado. Observatorio de la Economía Latinoamericana.

Ruso , J. A., Villamar , D. P., Ordeñana, A. R., \& Contreras, E. R. (2019). Análisis de la recaudación del régimen impositivo simplificado ecuatoriano (RISE) y su importancia en los ingresos tradicionales del Presupuesto General. INNOVA Research Journa, 4(3), 53-64.

Torgler, B., \& Schaltegger, C. A. (2005). Tax Morale and Fiscal Policy. Obtenido de https://www.econstor.eu/handle/10419/214344

Torres, V. (2012). La evasión tributaria: Marco conceptual de sus causas y medición. Alternativa Financiera(7), 45-58.

Washco, T. L. (2015). Análisis de las reformas tributarias e incidencia en la recaudación de los principales impuestos del Ecuador, 2009 - 2013. (Tesis de Maestría). Universidad de Cuenca. Cuenca, Ecuador.

Esta obra está bajo una Licencia Creative Commons

Attribución-NoCommercial 4.0 International

(cc) EY-NC 